\title{
Mechanical and Thermodynamic Properties of Half-metallic Ferromagnetism Containing Cobalt and Titanium
}

\author{
I. Asfour ${ }^{1,2, *}$, D. Rached ${ }^{1}$, Ababou Girard soraya ${ }^{3}$, Sébilleau Didier ${ }^{3}$ \\ ${ }^{1}$ Department of Physics, Faculty of Science, University Djillali Liabes, Algeria \\ ${ }^{2}$ Department of Material Technology, Faculty of Physics, University of Science and Technology-Mohamed-Boudiaf (USTO-MB), \\ Algeria \\ ${ }^{3}$ Department of Nanosciences Materials, Institute of Physics of Rennes, France
}

Copyright $\subseteq 2019$ by authors, all rights reserved. Authors agree that this article remains permanently open access under the terms of the Creative Commons Attribution License 4.0 International License

\begin{abstract}
We have studied the structural, electronic, elastic, magnetic and Thermodynamic properties of Co-based full-Heusler alloys $\mathrm{Co}_{2} \mathrm{TiSi}$ and $\mathrm{Co}_{2} \mathrm{TiGe}$. have been studied by first-principles full-potential linearized augmented plane wave (FPLAPW) method with the Generalized Gradient Approximation (GGA) based on density functional theory (DFT). This shows that the magnetic properties of the compound are dependent on electron concentration of main group element and all compunds are magnetic in their equilibrium L $2{ }_{1}$ structure. The electronic structure report that, our compounds have half-metallic (HM) nature. The mechanical results show that these compounds are mechanically stable. and exhibit $100 \%$ spin polarization at the Fermi level where it can be shifted within the energy-gap. In addition, the quasi-harmonic Debye model is applied to determine the thermal properties of the alloy.
\end{abstract}

Keywords Half-metallic, Heusler Alloys, Mechanical Properties, Thermodynamic Properties, Debye

\section{Introduction}

The history of a class of new materials can be traced back to the year 1903, Fritz Heusler alloy discovered that a type of formula $\mathrm{Cu}_{2} \mathrm{MnAl}$ behaves like a ferromagnetic material, although its components are not the magnetic materials in them same [1,2].Such ternary alloys are determined by the generic formulas $\mathrm{X}_{2} \mathrm{YZ}$ or XYZ [3,4], is characterized by the formula $X Y Z$ and called a semi-Heusler alloy. The -Heusler alloys $\left(\mathrm{X}_{2} \mathrm{YZ}\right)$ where $\mathrm{X}$ and $Y$ are transition metals, and $\mathrm{Z}$ is an element of group III, IV or $\mathrm{V}$, in some cases, $\mathrm{Y}$ is replaced by either a rare earth element. The $\mathrm{X}_{2} \mathrm{YZHeusler}$ compounds crystallize in the cubic L21 ( $\mathrm{AlCu}_{2} \mathrm{Mn}$-type) structure with the space group Fm-3m. In this structure, $\mathrm{X}, \mathrm{Y}$ and $\mathrm{Z}$ atoms are placed on the Wyckoff positions $8 \mathrm{c}(1 / 4,1 / 4,1 / 4), 4 \mathrm{a}(0,0,0)$ and $4 \mathrm{~b}$ $(1 / 2,1 / 2,1 / 2)$, respectively. The cubic $X_{2} Y Z$ compounds can also be found in theCuHg2Ti type structure. This formal XYXZ-type structure exhibits Td symmetry with the space group F-43m. In that structure the two $X$ atoms occupy nonequivalent positions in contrast to the $\mathrm{L} 22_{1}$ structure. This structure is frequently observed when the nuclearcharge of the $Y$ element is larger than the one of the $\mathrm{X}$ element from the same period, that is $\mathrm{Z}(\mathrm{Y})>\mathrm{Z}(\mathrm{X})$ for two 3 dtransition metals. In this structure, $X$ atoms occupy the nonequivalent $4 \mathrm{a}(0,0,0)$ and $4 \mathrm{c}(1 / 4,1 / 4,1 / 4)$ positions, while $\mathrm{Y}$ and $\mathrm{Z}$ atoms are located on $4 \mathrm{~b}(1 / 2,1 / 2$, $1 / 2)$ and $4 \mathrm{~d}(3 / 4,3 / 4,3 / 4)$ Wyckoff positions, respectively [5].

The two compounds $\mathrm{Co}_{2} \mathrm{CrSi}$ and $\mathrm{Co}_{2} \mathrm{CrGethese}$ are new innovative materials for many application spinotronique, these alloys have a special interest because of their relatively high Curie températeur. The two parents Heusler materials $\mathrm{Co}_{2} \mathrm{CrSi}$ and $\mathrm{Co}_{2} \mathrm{CrGe}$ proved to be very prospective materials for advanced thermoelectric applications such as in solid-state refrigeration and power generation. Heusler's materials are the subject of many studies. This type of material attracts the interest of chemists and physicists of the solid because of their remarkable physical and magnetic properties frequently encountered.Co-based Heusler alloys, which are considered promising candidates for commercial spintronics applications, in particular because of their high Curie temperatures.

\section{Calculation Methodology}

This study is performed using density functional theory DFT [6] calculations within the framework of full-potential 
linearized augmented plane-wave (FP-LAPW) method as implemented in WIEN2K package $[7,8]$. The generalized-gradient approximation (GGA) is used for the exchange correlation potential [9].The muffin-tin sphere radii RMTwere chosen as equal to $2.1,2.3,1.7$ and 2.2 a.u for the $\mathrm{Co}, \mathrm{Ti}$,Siand $\mathrm{Ge}$ atoms, respectively.The plane wave cut-off parameter is taken as RMT*Kmax=9, TheBrillouin zone sampling was performed according to the Monkhorst-Pack scheme and for k-space integration, a (14 * $14 * 14$ ) mesh was used resulting in 104k points of the irreducible part in the Brillouin zone [7].Study of thermodynamic properties was performed within the quasi-harmonic Debye model implemented in the Gibbs program [8]. The quasi-harmonic Debye model allows us to obtain all thermodynamics quantities from the calculated energy-volume points. Detailed descriptions of this procedure can be found in Refs. [8-12].

\section{Results and Discussion}

\subsection{Structural Properties}

To obtain the equilibrium lattice constant and determine the stable structure of these alloys, we perform structural optimizations on $\mathrm{Co}_{2} \mathrm{TiSi}$ and $\mathrm{Co}_{2} \mathrm{TiGe}$ alloys for nonmagnetic (NM), magnetic (M) and configurations for both $\mathrm{AlCu}_{2} \mathrm{Mn}$-type (L21) and $\mathrm{CuHg}_{2}$ Ti-type structures. It is clearly seen that these structures are more stable in the $\mathrm{AlCu}_{2} \mathrm{Mn}$-type (L21) than in the $\mathrm{CuHg}_{2}$ Ti-type (see Fig. 1). The calculated total energies versus volume are fitted to the

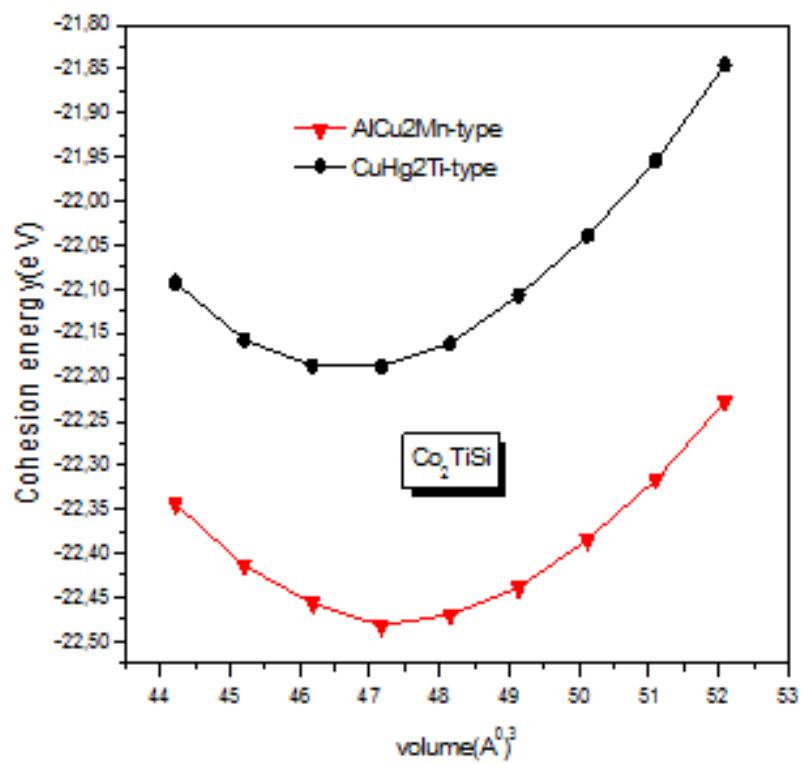

empirical Murnaghan's equation of state [13] to determine the ground state properties. For $\mathrm{Co}_{2} \mathrm{TiSi}$ in the $\mathrm{AlCu}_{2} \mathrm{Mn}$-type structure

\subsection{Electronic Properties}

To elucidate the nature of the electronic band structure, we also calculated the total and partial densities of states, as shown in Figures 2 most transport properties are determined on the basis knowledge of the density of states.Generaly, in the vicinity of the Fermi level, the bands are mainly due to orbital states $\mathrm{d}$ of $\mathrm{Ti}$ and the transition metals; this is justified by the half metallic character.

Densities of states confirming the metallic character for the majority of densities are presented in Figure 2. A lack of electronic states at the Fermi level of minority spins us closer to the semiconductor and semi-metallic character for both alloys.From figures 3 , it is clear that there are three distinct regions in the spin-up state and in the spin-down state separated by gaps materials.

We notice an overlap valence and conduction bands to the state spin-up, while the existence of electronic states at the Fermi level tells us about the nature of these metal alloys. The presence of electronic states is most obvious for the spin up, it is minimal for the spin down and shows the near character semiconductor, in this state we see a gap between the conduction and the valence band. It can be said that the compounds and their alloys are semiconductors in the state spin-dn. This means that the system has a half-metallic character. We notice a change in indirect gap for ternary alloy materials studied

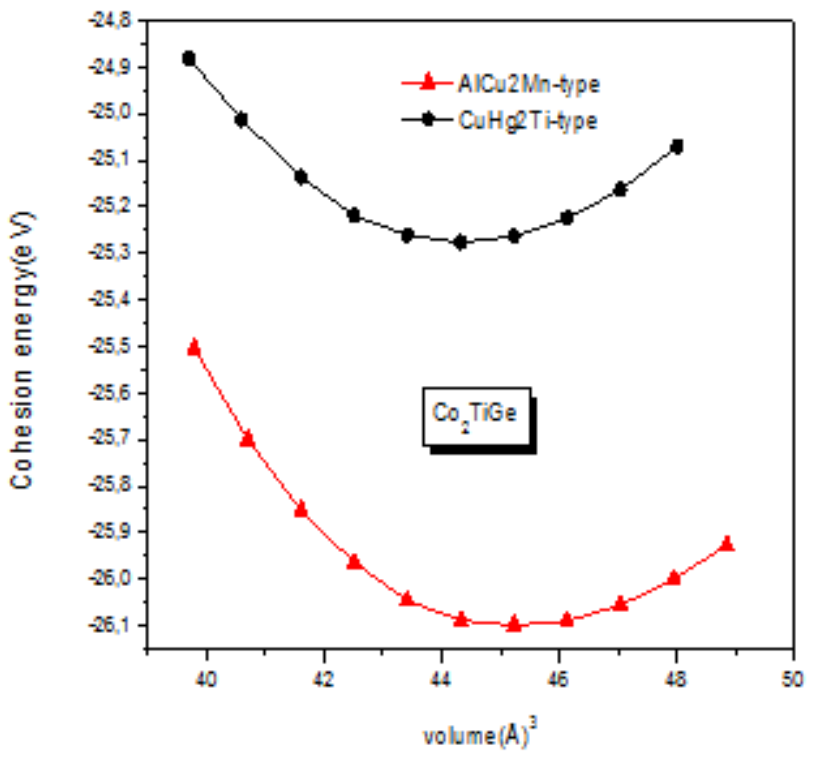

Figure 1. Calculated total energy versus volume curves ferromagnetic (FM) state for $\mathrm{AlCu}_{2} \mathrm{Mn}$-type and $\mathrm{CuHg}_{2} \mathrm{Ti}_{\text {-type }}$ phases of $\mathrm{Co}_{2} \mathrm{TiSi}$ and $\mathrm{Co}_{2} \mathrm{TiGe}$ 

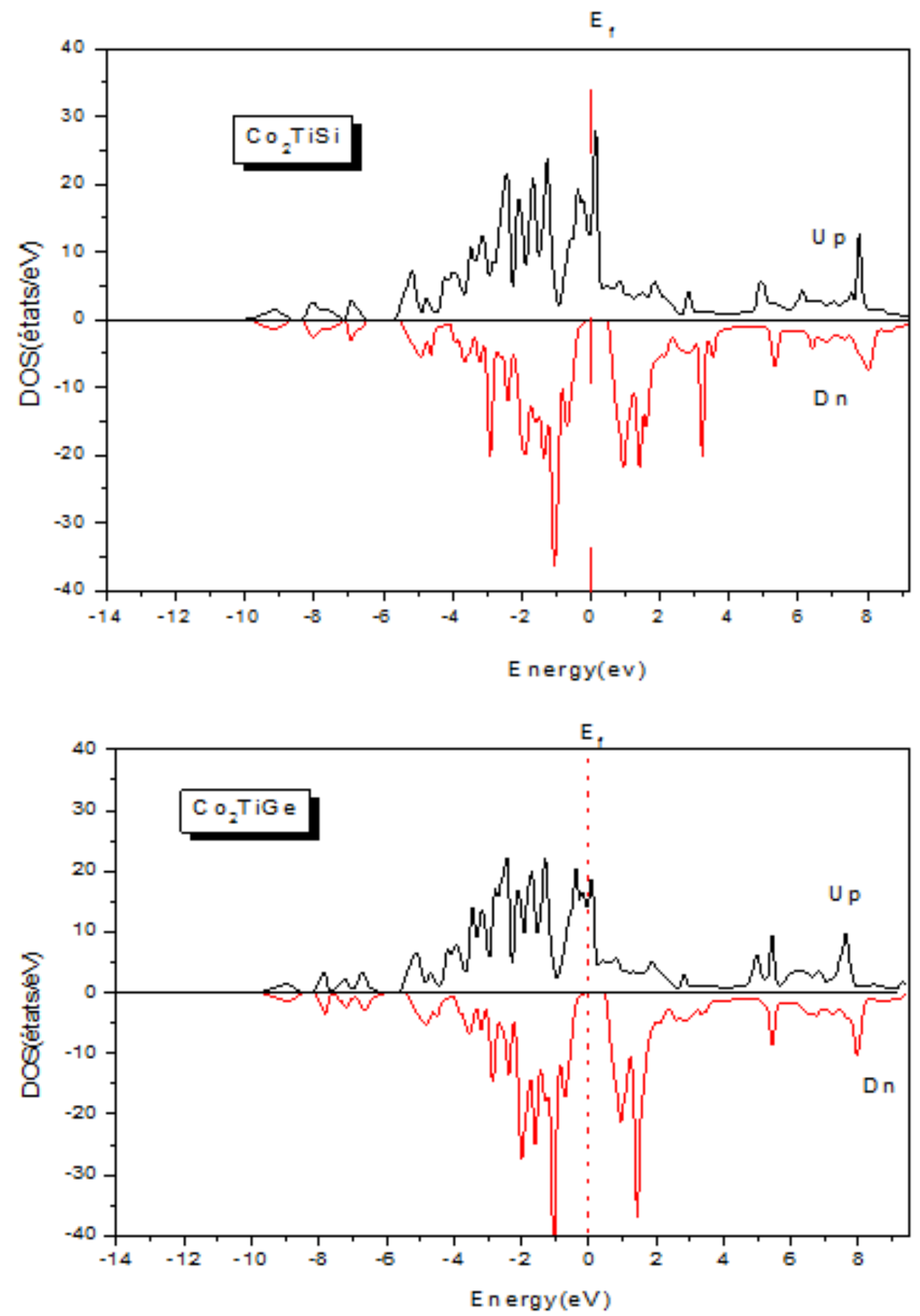

Figure 2. Total and partial density of states of the two ternary Heusler alloys $\mathrm{Co}_{2} \mathrm{TiGe}$ and $\mathrm{Co}_{2} \mathrm{TiGe}$. 

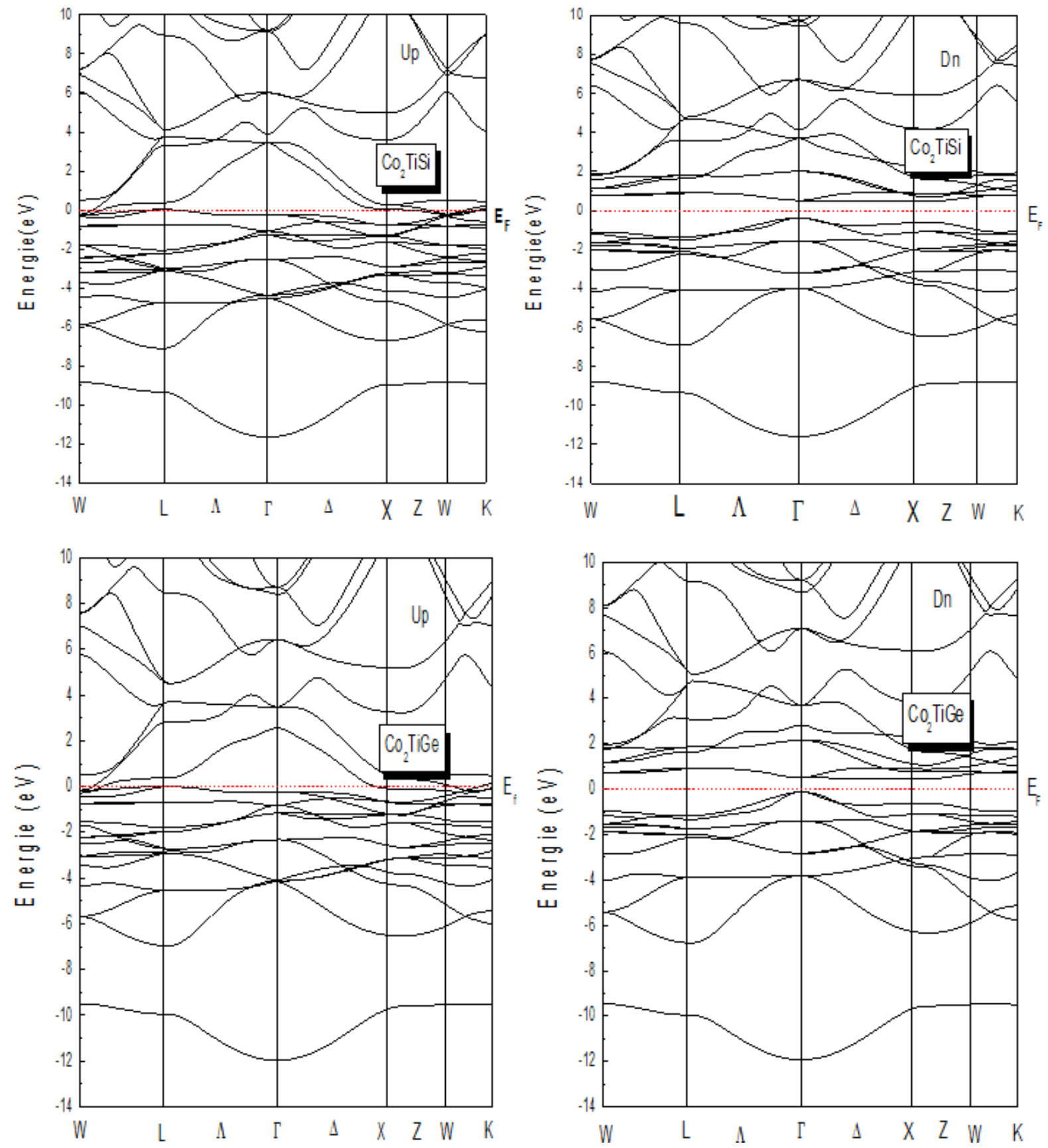

Figure 3. Calculated band structures of the two ternary Heusler alloy $\mathrm{Co}_{2} \mathrm{TiSi}$ and $\mathrm{Co}_{2} \mathrm{TiGe}$.

\subsection{Magnetic Properties}

The term half-metallicity was introduced for the first time by Groot et al[14], focusing on the calculation of the half-Heusler alloy band structure: NiMnSb[15]. Conventional ferromagnetic materials have an electronic density of statesN (EF)) at the Fermi level for the majority spin electrons (up: $\mathrm{N} \uparrow(\mathrm{EF})$ ) and minority spin (down: $\mathrm{N} \downarrow$ (EF)). We can then define the polarization $\mathrm{P}$ in spin, which measures the spin asymmetry, by the expression[16]:

$$
P=\frac{N_{\downarrow}\left(E_{F}\right)-N_{\downarrow}\left(E_{F}\right)}{N_{\downarrow}\left(E_{F}\right)+N_{\downarrow}\left(E_{F}\right)}
$$

The magnetic moment per formula unit for the alloys of $3 \mathrm{~d}$ elements can be determined from the number of their valence electrons using Slater-Pauling (SP) rule [17].According to this rule, magnetic moment for the half metallic moment for the half metallic full-Heusler alloy is given by $\mathrm{Mt}=\mathrm{Zt}-24$, where $\mathrm{Mt}$ is the total magnetic moment per unit cell and $\mathrm{Zt}$ is the total number of valence electrons. The magnetic moment obtained by present calculations is $4.0075 \mu_{\mathrm{B}}$ and $4.0020 \mu_{\mathrm{B}}$ for $\mathrm{Co}_{2} \mathrm{TiSi}$ and $\mathrm{Co}_{2} \mathrm{TiGe}$ respectively which agrees well with values from SlaterPauling rule [17] as well as other calculations [18-20]. The energy band structure of a semi-metallic material has an asymmetry between the spin up states and spins down with 
a gap or a pseudo energy gap at the Fermi level. This gives rise to the polarizations of the conduction electrons at the Fermi level which may reach $100 \%$.

\subsection{Thermal Properties}

We can calculate the thermodynamic quantities for the Two Heusler alloysCo2TiSi and Co2TiGe through the Debye model quasi-harmonic implemented in the Gibbs program [21.22] in which the non-equilibrium Gibbs function $\mathrm{G}^{*}(\mathrm{~V}, \mathrm{P}, \mathrm{T})$.

Through the quasi-harmonic Debye model, one could compute the thermodynamic quantities of any temperatures and pressures of the ternary Heusler alloys Co2TiSi and Co2TiGe from the calculated $\mathrm{E}-\mathrm{V}$ data at $\mathrm{T}=0$ and $\mathrm{P}=0$. ( $\mathrm{T}$ being the temperature and $\mathrm{P}$ the pressure).

The relationship between the lattice parameter and the temperature at different pressures is shown in Figures 4-5 the lattice parameter increases to a very moderate with temperature. On the other hand, it is noted in Figures 6-7 that the relationship between the bulk modulus and the pressure is virtually linear. The bulk modulus increases with pressure and decreases with temperature.

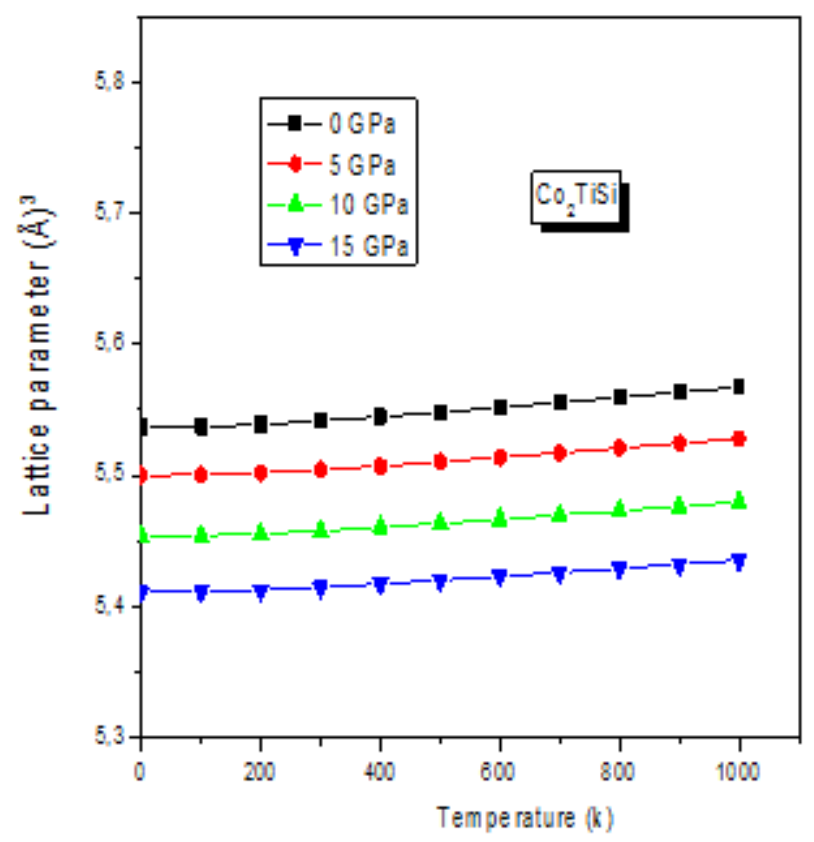

Figure 4. Variation of the lattice parameter as a function of temperature and pressureinCo $\mathrm{Co}_{2}$ TiSialloy.

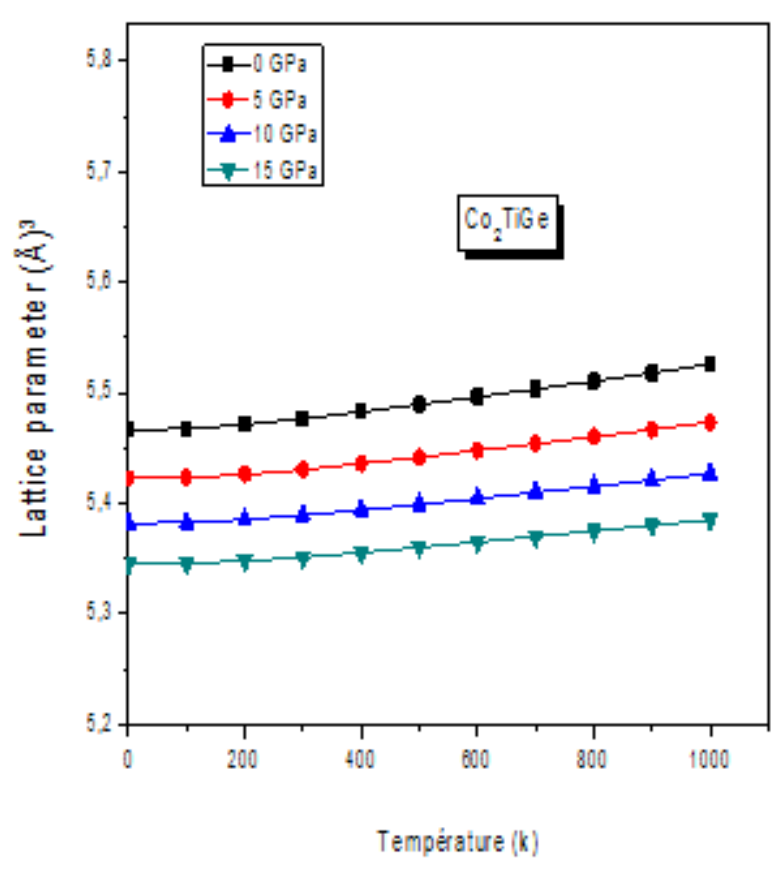

Figure 5. Variation of the lattice parameter as a function of temperature and pressure in $\mathrm{Co}_{2} \mathrm{TiGe}$ alloy.

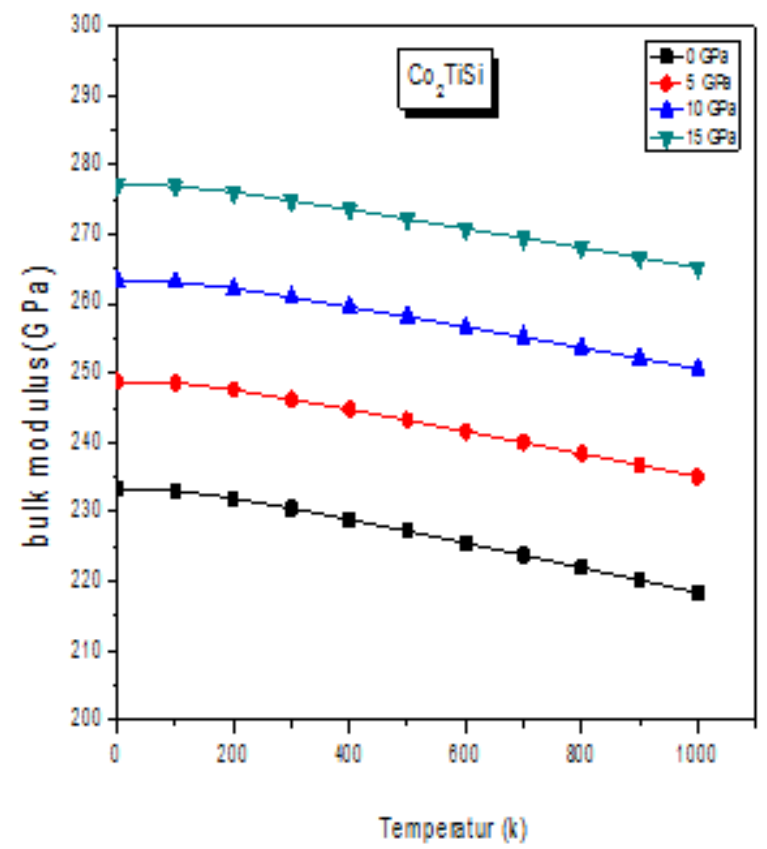

Figure 6. Variation of the bulk modulus as a function of temperature and pressureinCo $\mathrm{CiSi}_{2}$ alloy alloy. 


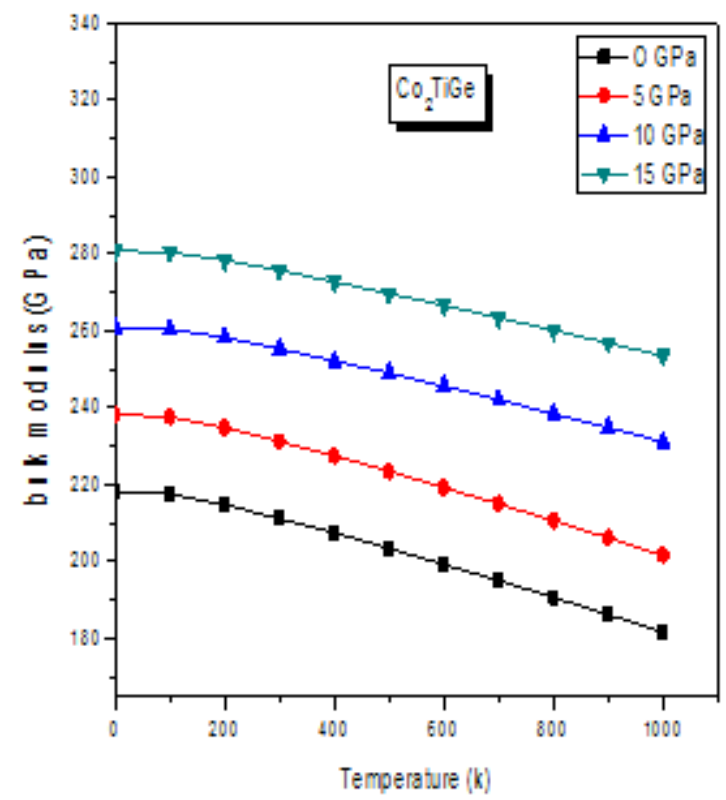

Figure 7. Variation of the bulk modulus as a function of temperature and pressure in $\mathrm{Co}_{2}$ TiGealloy .

The investigation on the heat capacity of crystals is an old topic of the condensed matter physics with which illustrious names are associated. Knowledge of the heat capacity of a substance not only provides essential insight into its vibrational properties but is also mandatory for many applications. Two famous limiting cases are correctly predicted by the standard elastic continuum theory [23]. At high temperatures, the constant-volume heat capacity $\mathrm{Cv}$ tends to the Petit and Dulong limit [24],the Figures 8 and 9 show the variation of the heat capacity Cvcontant volume depending on the temperature for different pressures. This quantity indicates a strong increase up to $\sim 500 \mathrm{~K}$, which is due to anharmonic approximation Debye model. Values are purely predictive since we have no experimental data. However, at higher temperatures and at higher pressures, the effect of anharmonic $\mathrm{Cv}$ is deleted, and $\mathrm{Cv}$ tends towards the limit of Dulong-Pettit. $(\mathrm{CV}$ $\cong 74.60 \mathrm{~J} . \mathrm{mol}-1 . \mathrm{K}-1-74.46 \mathrm{~J} \cdot \mathrm{mol}-1 . \mathrm{K}-1$, respectively $\mathrm{Co} 2 \mathrm{CrAl}$ and $\mathrm{Co} 2 \mathrm{CrGa}$ is shown in figure 8-9.

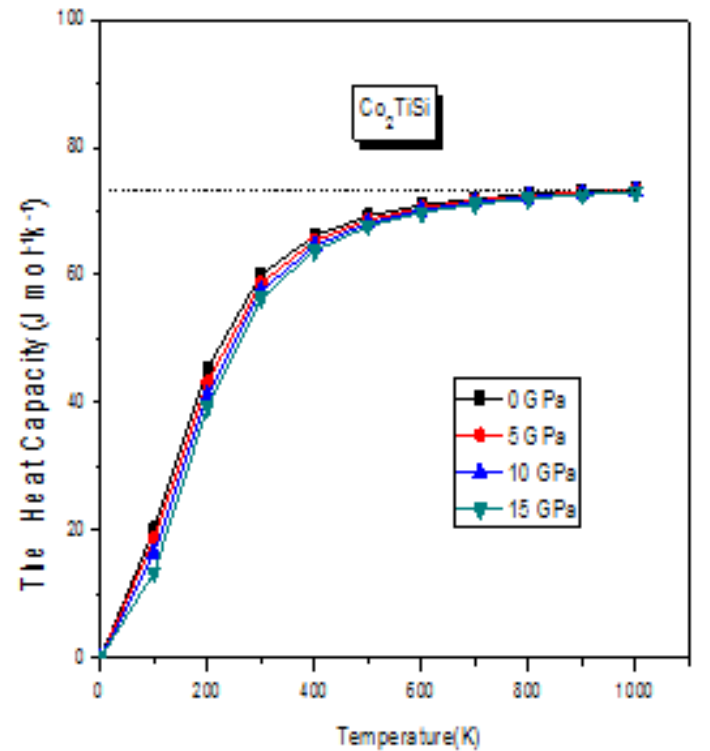

Figure 8. Variation of the heat capacity as a function of temperature and pressurein $\mathrm{Co}_{2} \mathrm{TiSi}$ alloy.

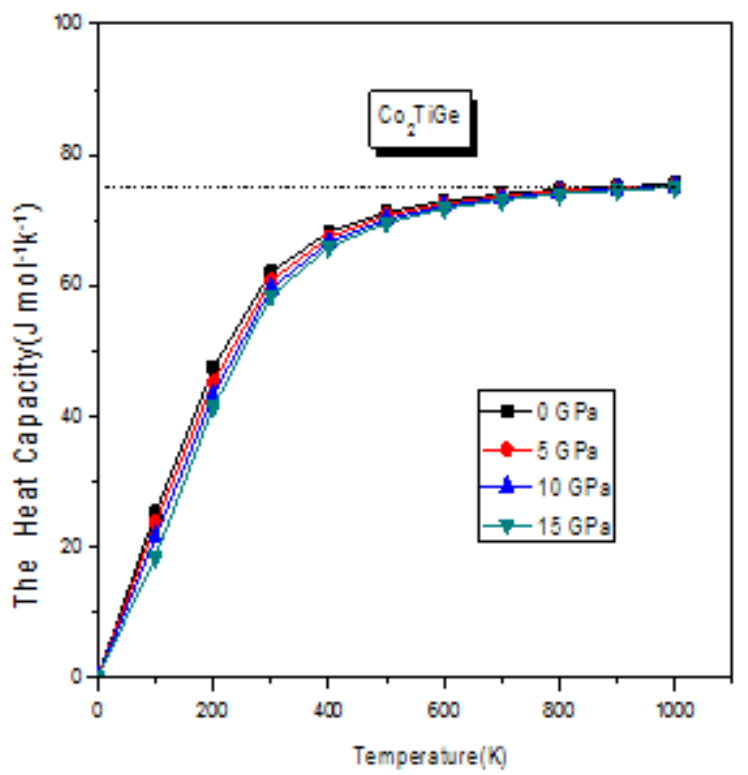

Figure 9. Variation of the heat capacity as a function of temperature and pressureinCo $\mathrm{Co}_{2} \mathrm{TiGe}$ alloy. 
In figures 10 and 11 , we present the variation of the Debye temperature $\theta \mathrm{D}$ as a function of temperature and pressure, respectively, one can observe that $\theta \mathrm{D}$ is nearly constant from 0 to $100 \mathrm{~K}$ and decreases linearly with increasing temperature from $\mathrm{T}>200 \mathrm{~K}$. It can be seen that the Debye temperature $\theta \mathrm{D}$ increases with pressure and decreases with temperature.

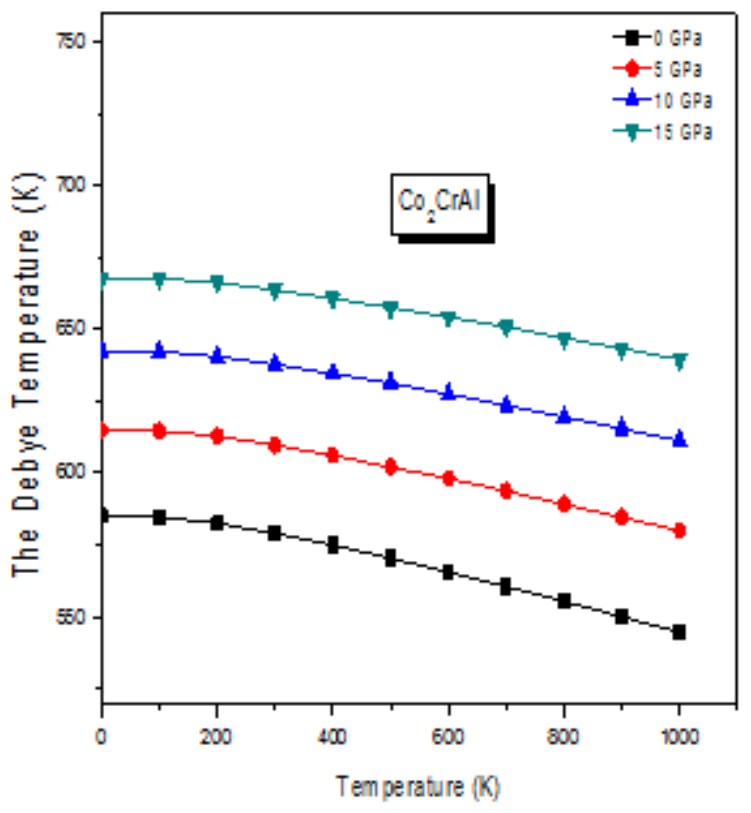

Figure 10. The Debye temperature $\theta_{D}$ as a function of temperature and pressure in $\mathrm{Co}_{2} \mathrm{TiSi}$ alloy.

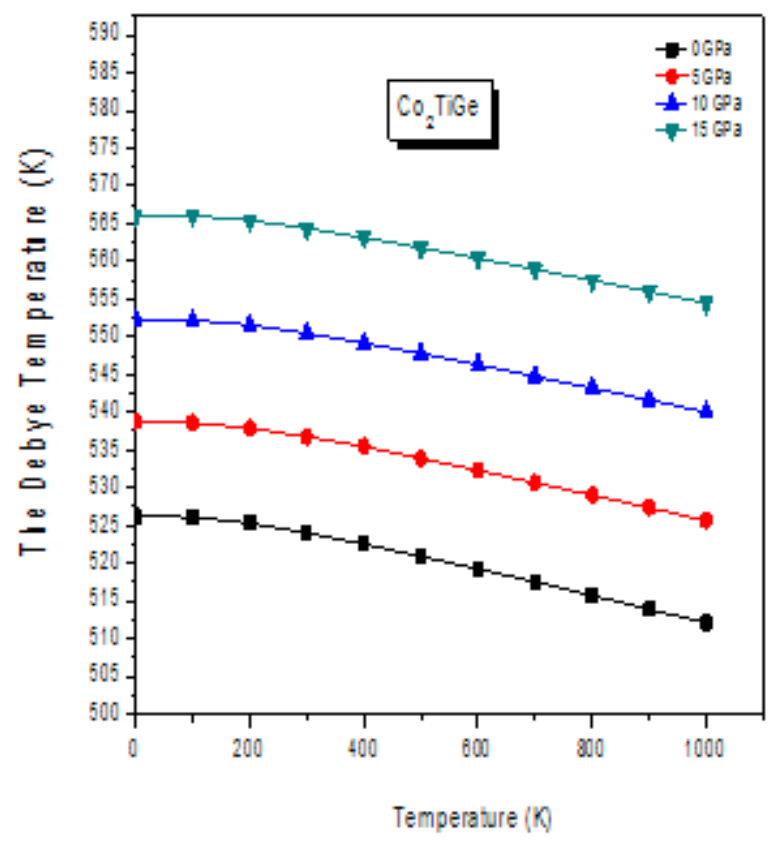

Figure 11. The Debye temperature $\theta_{D}$ as a function of temperature and pressure in $\mathrm{Co}_{2} \mathrm{TiGe}$ alloy.

The coefficient of thermal expansion $\alpha$ has been predicted Figur.12-13, He quickly believes in cube power of temperature then tends towards a limit.
We note, for a given pressure $\alpha$ increases with temperature when $\mathrm{T} \leqslant 300 \mathrm{~K}$ (at low temperature), especially at zero pressure, and tends gradually to increase linearly at higher temperatures. As the pressure increases, the variation of a with the temperature becomes smaller. For a given temperature, $\alpha$ decrease sharply with increasing pressure, and is very low at higher temperatures and higher pressures as well.

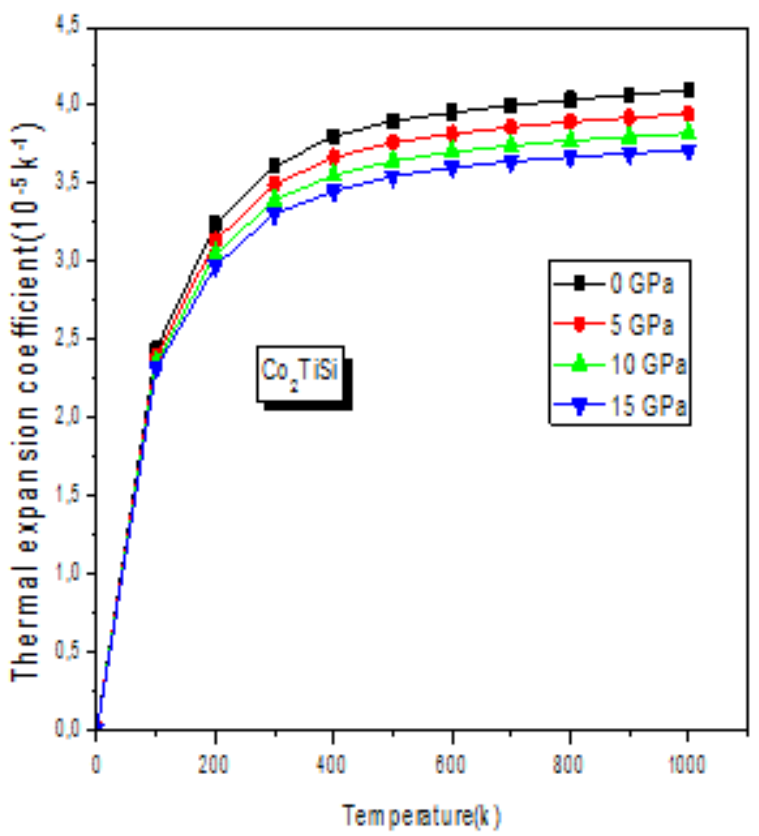

Figure 12. Variation temperature coefficient as a function of temperature for different pressures for $\mathrm{Co}_{2} \mathrm{TiSi}$

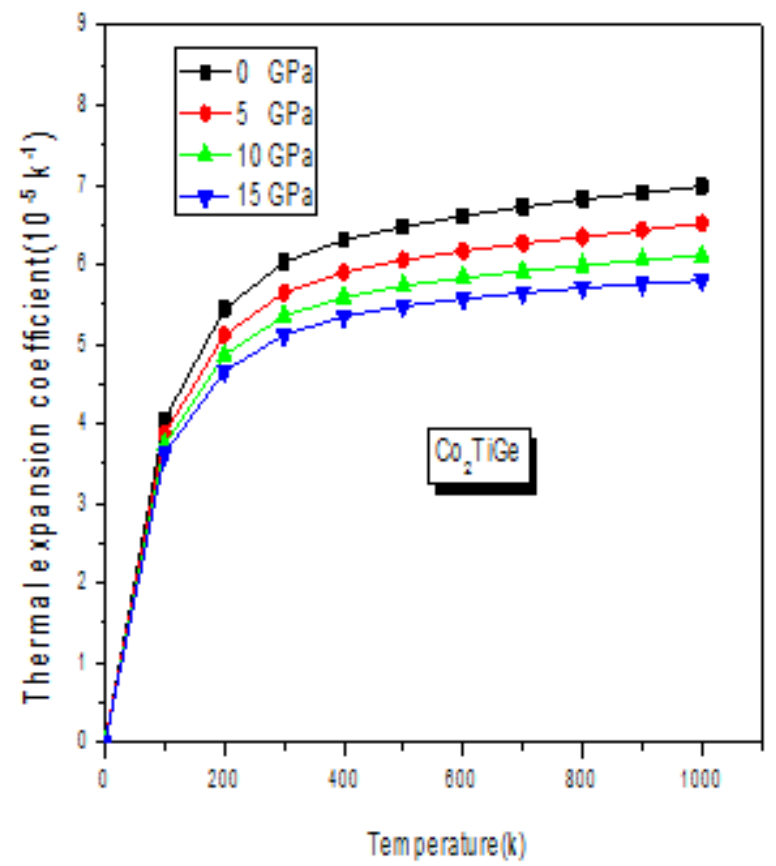

Figure 13. Variation temperature coefficient as a function of temperature for different pressures for $\mathrm{Co}_{2} \mathrm{TiGe}$ 


\subsection{Mechanical Properties}

The elastic constants are also an important parameters for describing the mechanical properties of materials that undergo stress, and give important information concerning the binding characteristic between adjacent atomic planes, anisotropic character of binding and structural stability. There are independent elastic constants, $\mathrm{C}_{i j}$, but the symmetry of cubic crystal reduces this number to only three independent elastic constants $\mathrm{C}_{11}, \mathrm{C}_{12}$ and $\mathrm{C}_{44}$

In Table 1, the calculated three independent elastic constants $\mathrm{C}_{11}, \mathrm{C}_{12}$ and $\mathrm{C}_{44}$, shear modulus $\mathrm{G}$, ratio of $\mathrm{B} / \mathrm{G}$, Young's modulus E, Poisson's ratio (m), Zener anisotropy factor (A), density (q), longitudinal elastic wave velocities $\mathrm{vl}$, transverse elastic wave velocities vt, average acoustic velocity vm, and Debye temperature $(\mathrm{hD})$ of $\mathrm{Co}_{2}$ TiSiand $\mathrm{Co}_{2} \mathrm{TiGe}$ for various compositions are listed. To our knowledge, we note that no result regarding the elastic constants are so far available in the literature. Therefore, our results are considered as purely predictive.

The traditional mechanical stability conditions in cubic crystals at equilibrium are expressed in terms of elastic constants as follows: $\mathrm{C}_{11}-\mathrm{C}_{12}>0, \mathrm{C}_{44}>0, \mathrm{C}_{11}+2 \mathrm{C}_{12}>0$ and $\mathrm{C}_{12}<\mathrm{B}<\mathrm{C}_{11}$ [25].

The computed elastic constants satisfy the above stability criteria, indicating that these compounds are elastically stable.

In order to predict the brittle and ductile behavior of materials, Pugh [26] proposed an approximate criterion by the ratio of $\mathrm{B} / \mathrm{G}$. Higher (lower) $\mathrm{B} / \mathrm{G}$ ratio corresponds to ductile (brittle) behavior, and the critical value that separates brittle and ductile materials is about 1.75 . The data in the Table 1 indicate that the $\mathrm{B} / \mathrm{G}$ ratio is 3.3894 and 3.1245 suggesting that $\mathrm{Co}_{2} \mathrm{TiSi}$ and $\mathrm{Co}_{2} \mathrm{TiGeHeusler} \mathrm{alloys}$ are ductile.

The Young's modulus E and Poisson's ratio $\mathrm{m}$ are important in technological and engineering application [27]. Young's modulus is defined as the ratio of stress and strain when Hooke's law holds. The Young's modulus of a material is the usual property used to characterize stiffness. The higher the value of E, the stiffer is the material. From Table 1.

The value of the Poisson's ratio is indicative of the degree of directionality of the covalent bonds. Our obtained values for Poisson's ratio is 0.3727 and 0.3787 , which is an indication that the interatomic forces are central forces [28]. From our calculated values of the Zener anisotropy factor $\mathrm{A}$, which is a measure of the degree of elastic anisotropy of the crystal, we note that our Heusler alloys are elastically isotropic.

The Debye temperature is known to be an important fundamental parameter closely related to many physical properties, such as specific heat and melting temperature. At low temperatures, the vibrational excitations arise solely from acoustic vibrations. Hence, at low temperatures the Debye temperature calculated from elastic constants is the same as that determined from specific heat measurements
Table 1. Calculated lattice constant (a), bulk modulus (B), pressure derivative of the bulk modulus ( $\left.\mathrm{B}^{\prime}\right)$, elastic constants $\left(\mathrm{C}_{i j}\right)$, shear modulus $(\mathrm{G})$, ratio of $\mathrm{B} / \mathrm{G}$, Young's modulus $(\mathrm{E})$, Poisson'sratio $(v)$, zener anisotropy factor (A), density (q), longitudinal elastic wave velocities $\left(\mathrm{V}_{l}\right)$, transverse elastic wave velocities $\left(\mathrm{V}_{t}\right)$, average acoustic velocity $\left(\mathrm{V}_{m}\right)$, and Debyetemperature $\left(\theta_{\mathrm{D}}\right)$ of $\mathrm{Co}_{2}$ TiSiand $\mathrm{Co}_{2} \mathrm{TiGe}$ for various compositions.

\begin{tabular}{|c|c|c|}
\hline & $\mathrm{Co}_{2} \mathrm{TiSi}$ & $\mathrm{Co}_{2} \mathrm{TiGe}^{2}$ \\
\hline $\mathrm{a}(\AA)$ & 5.7045 & 5.7816 \\
$\mathrm{a}_{\mathrm{exp}}(\AA)$ & $5.733^{\mathrm{a}}$ & $5.819^{\mathrm{a}}$ \\
$\mathrm{a}_{\mathrm{calc}}(\AA)$ & $5.758^{\mathrm{a}}$ & $5.850^{\mathrm{a}}$ \\
\hline $\mathrm{B}(\mathrm{Gpa})$ & 244,8304 & 237.6002 \\
\hline $\mathrm{B}^{\prime}$ & 4.5151 & 4.1277 \\
$\mathrm{C}_{11}(\mathrm{GPa})$ & 234.1536 & 232.2598 \\
\hline $\mathrm{C}_{12}(\mathrm{GPa})$ & 150.0187 & 145.8203 \\
\hline $\mathrm{C}_{44}(\mathrm{GPa})$ & 54.1728 & 55.2045 \\
\hline $\mathrm{G}(\mathrm{Gpa})$ & 54.1827 & 49.4786 \\
\hline $\mathrm{B} / \mathrm{G}$ & 3.3894 & 3.1245 \\
\hline $\mathrm{E}(\mathrm{GPa})$ & 138.0081 & 0.3987 \\
\hline$v$ & 0.3827 & 0.9782 \\
\hline $\mathrm{A}$ & 0.9987 & 4.0132 \\
\hline$\left.\rho_{(\mathrm{g} / \mathrm{cm}}^{3}\right)$ & 4.7845 & 6852.3874 \\
\hline $\mathrm{V}_{\mathrm{l}}(\mathrm{m} / \mathrm{s})$ & 7030.3996 & 3784.8853 \\
\hline $\mathrm{V}_{\mathrm{t}}(\mathrm{m} / \mathrm{s})$ & 3258.2705 & 3458.7537 \\
\hline $\mathrm{V}_{\mathrm{m}}(\mathrm{m} / \mathrm{s})$ & 3781.5095 & 325.7219 \\
\hline$\theta_{\mathrm{D}}(\mathrm{K})$ & 385.5389 & \\
\hline & & \\
\hline
\end{tabular}

${ }^{\mathrm{a}}$ Ref.[29]

\section{Conclusions}

The main investigation's goal of this work is to give a report onthe structural, mechanical, magneto-electronic and thermodynamic properties of the two ternary Heusler alloys $\mathrm{Co}_{2} \mathrm{TiSi}$ and $\mathrm{Co}_{2} \mathrm{TiGe}$ at ambient as well as at elevated temperatures. With the linearized augmented plane wave method based on density functional theory and implemented in wien $2 \mathrm{k}$ code. For exchange correlation potential we have used the generalized gradient approximation (GGA) of Perdew et al. Our interest in this study was justified by the fact that the properties of these compounds are not available in the literature. The choice of compounds was warranted by the great deal of attention given to these Heusler alloys because of their large field of applications. The electronic band structures show a halfmetallic- character. The estimated elastic constants confirm the mechanical stability of our compounds. The quasi-harmonic Debye model is success-fully applied to determine the thermal properties at different temperatures and pressures. finally, we can report that due to the potential half-metallicity exhibited by our investigated alloys, these compounds could be used for spintronic application..

\section{REFERENCES}

[1] F. Heusler,W. Starck, E. Haupt, VerhDPG 5 (1903) 220. 
[2] F. Heusler, VerhDPG 5(1903) 219.

[3] M. Parsons, J. Grandle, B. Dennis, K. Neumann, K. Ziebeck, J. Magn. Magn. Mater. 185 (1995) 140.

[4] E.P. Wohlfahrth, K.H.J. Bushow, Ferromagnetic Materials, vol. 4, Elsevier, Amsterdam, 1998.

[5] H.C. Kandpal, G.H. Fecher, C. Felser, J. Phys. D: Appl. Phys. 40 (2007) 1507.

[6] W. Kohn, L.J. Sham, Physical Review 140 (1965) A1133

[7] M. Petersen, F. Wagner, L. Hufnagel, M. Scheffler, P. Blaha, K. Schwarz,Computer Physics Communications 126 (2000) 294.

[8] S.E. Kulkova, S.S. Kulkov, A.V. Subashiev, Computational Materials Science 36 (2006)249

[9] M.A. Blanco, A. Martín Pendás, E. Francisco, J.M. Recio, R. Franco, J. Mol. Struct.Theochem. 368 (1996) 245.

[10] M. Flórez, J.M. Recio, E. Francisco, M.A. Blanco, A. Martín Pendás, Phys. Rev. B 66(2002)144112.

[11] I. Asfour, H. Rached, S. Benalia, D. Rached, J. Alloy. Comp. 676 (2016) 440-451.

[12] E. Francisco, M.A. Blanco, G. Sanjurjo, Phys. Rev. B 63 (2001) 094107.

[13] F.D. Murnaghan, Proceedings of the National Academy of Sciences of the United States of America 30, 5390,(1944)

[14] H.C. Kandpal, G.H. Fecher, C. Felser, J. Phys. D: Appl. Phys. 40 (2007) 1507.

[15] I. Asfour, H.Rached, D. Rached, M. Caid, M. Labair, J. Alloy. Comp. 742 (2018) 736-750

[16] I. Asfour, D.Rached,Universal Journal of Mechanical
Engineering 6(2) 21-37,2018.

[17] H.C. Kandpal, G.H. Fecher, C. Felser, J. Phys. D: Appl. Phys. 40 (2007) 1507.

[18] L. Heyne, T. Igarashi, T. Kanomata, K.U. Neumann, B. Ouladdiaf, K.R.A. Ziebeck, J PhysCondens Matter 17 (2005) 4991.

[19] I. Asfour, D.Rached,Universal Journal of Mechanical Engineering 6(2) 21-37,2018.

[20] S.E. Kulkova, S.S. Kulkov, A.V. Subashiev, Computational Materials Science 36 (2006)249

[21] E. Francisco, M.A. Blanco, G. Sanjurjo, Phys. Rev. B 63 (2001) 094107.

[22] J.P. Poirier, Introduction to the Physics of the Earth's Interior, Cambridge University Press, Oxford, 2000, p. 39.

[23] R. Hill, Proc. Phys. Soc. A 65 (1952) 349.

[24] E. Francisco, M.A. Blanco, G. Sanjurjo, Phys. Rev. B $63,094107,(2001)$.

[25] J.F. Nye, Physical Properties of Crystals, Oxford University Press, 1985.

[26] S.F. Pugh, Philos. Mag. 45 (1954) 823.

[27] F. Peng, D. Chen, X.D. Yang, Solid State Commun. 149 (2009) 2135.

[28] F. Chu, Y. He, D.J. Thome, T.E. Mitchell, Scr. Metall. Mater.33 (1995) 1295.

[29] J.Barth,G. H. Fecher, B. Balke, T.Graf, A. Shkabko, A Weidenkaff, P . Klaer, M. Kallmayer, H.-J. Elmers, H. Yoshikawa, S. Ueda, K. Kobayashi, and C.Felser, Philos. Trans. Roy. Soc. A369,3588-3601(2011). 